\title{
Three Integral Representations for the Trigamma Function and Some Special Identities
}

\section{Edigles Guedes ${ }^{1}$ and Prof. Dr. K. Raja Rama Gandhi ${ }^{2}$ \\ Number Theorist, Brazil ${ }^{1}$}

Resource perosn in Mathematics for Oxford University Press and Professor at BITS-Vizag ${ }^{2}$

Abstract. I proved three new integral representations for the trigamma function.

\section{INTRODUCTION}

Utilizing a classical integral representation and unorthodox method of substitution, I demonstrated that:

$$
\psi_{1}(s)=\int_{0}^{\infty} \frac{(u+1)^{-s} \ln (u+1)}{u} d u
$$

and

$$
\psi_{1}(s)=\int_{0}^{\infty} \frac{t e^{(1-s) t}}{e^{t}-1} d t
$$

as well as proved, using an integral representation of the natural logarithm function, that:

$$
\psi_{1}(s)=\int_{0}^{1} \int_{0}^{1} \frac{x^{s-1}}{y(\ln y-1)(\ln y-x)} d x d y .
$$

\section{THEOREM}

Theorem 1. For $\mathfrak{R}(s)>0$, then

$$
\psi_{1}(s)=\int_{0}^{\infty} \frac{(u+1)^{-s} \ln (u+1)}{u} d u,
$$

where $\psi_{1}(s)$ denotes the trigamma function and $\ln s$ denotes the natural logarithm.

Proof. In [1], the trigamma function is defined by

$$
\psi_{1}(s):=-\int_{0}^{1} \frac{t^{s-1}}{1-t} \ln t d t .
$$

Take $t=\frac{a+b u}{1+u}$ and $d t=\left[\frac{b}{1+u}-\frac{a+b u}{(1+u)^{2}}\right] d u$ in (2), I obtain

$$
\psi_{1}(s)=-\int_{-\frac{a}{b}}^{-\frac{a-1}{b-1}}\left[\frac{\left(\frac{a+b u}{1+u}\right)^{s-1}}{1-\frac{a+b u}{1+u}} \ln \left(\frac{a+b u}{1+u}\right)\right]\left[\frac{b}{1+u}-\frac{a+b u}{(1+u)^{2}}\right] d u,
$$

Let $-\frac{a-1}{b-1}=\tan (\pi \theta)$ and $-\frac{a}{b}=\cot (\pi \theta)$, therefore,

$$
a=\frac{(\tan (\pi \theta)+1) \cot (\pi \theta)}{\cot (\pi \theta)-\tan (\pi \theta)}, \quad b=-\frac{\tan (\pi \theta)+1}{\cot (\pi \theta)-\tan (\pi \theta)} .
$$

Put (4) in (3) and simplifying, I have

$$
\psi_{1}(s)=-\int_{\cot (\pi \theta)}^{\tan (\pi \theta)} f(u, \theta) d u,
$$


where

$$
f(u, \theta)=\frac{2 \cot (2 \pi \theta)\left(\frac{\cos (\pi \theta)-u \sin (\pi \theta)}{(u+1)(\cos (\pi \theta)-\sin (\pi \theta))}\right)^{s} \ln \left(\frac{\cos (\pi \theta)-u \sin (\pi \theta)}{(u+1)(\cos (\pi \theta)-\sin (\pi \theta))}\right)}{(u-\tan (\pi \theta))(u-\cot (\pi \theta))} .
$$

Consider the limit $\theta \rightarrow 1$ in (5)

$$
\lim _{\theta \rightarrow 1} \psi_{1}(s)=-\lim _{\theta \rightarrow 1} \int_{\cot (\pi \theta)}^{\tan (\pi \theta)} f(u, \theta) d u,
$$

then

$$
\begin{gathered}
\lim _{\theta \rightarrow 1} \psi_{1}(s)=\psi_{1}(s), \\
\lim _{\theta \rightarrow 1} f(u, \theta)=-\frac{\left(\frac{1}{u+1}\right)^{s} \ln \left(\frac{1}{u+1}\right)}{u}, \\
\lim _{\theta \rightarrow 1} \tan (\pi \theta)=0, \\
\lim _{\theta \rightarrow 1} \cot (\pi \theta)=\infty .
\end{gathered}
$$

Hence, from (6) and (7), it follows that

$$
\psi_{1}(s)=\int_{0}^{\infty} \frac{(u+1)^{-s} \ln (u+1)}{u} d u
$$

Corollary 1. For $\mathfrak{R}(s)>0$, then

$$
\psi_{1}(s)=\int_{0}^{\infty} \frac{t e^{(1-s) t}}{e^{t}-1} d t
$$

Proof. I substitute $u+1=e^{t}$ and $d u=e^{t} d t$ in Theorem 1 .

3.1.11. THEOREM. For $\mathfrak{R}(s)>0$, then

$$
\psi_{1}(s)=\int_{0}^{1} \int_{0}^{1} \frac{x^{s-1}}{y(\ln y-1)(\ln y-x)} d x d y,
$$

where $\psi_{1}(z)$ denotes the trigamma function and $\ln s$ denotes the natural logarithm.

Proof. In [2], I prove that

$$
\frac{\ln x}{x-1}=\int_{0}^{1} \frac{1}{y(\ln y-x)(\ln y-1)} d y,
$$

and I know that trigamma function is defined by

$$
\psi_{1}(s) \stackrel{\text { def }}{=}-\int_{0}^{1} \frac{x^{s-1} \ln x}{1-x} d x .
$$

Substituing (9) in (10), then,

$$
\psi_{1}(s)=\int_{0}^{1} \int_{0}^{1} \frac{x^{s-1}}{y(\ln y-1)(\ln y-x)} d x d y .
$$


Special Identities. Utilizing the Corollary 1, I calculate

$$
\begin{aligned}
& \psi_{1}\left(\frac{1}{3}\right)=\frac{4 \pi^{2}}{3}-\psi_{1}\left(\frac{2}{3}\right) \\
& \psi_{1}\left(\frac{1}{4}\right)=\frac{G_{3,3}^{3,2}\left(1 \mid \begin{array}{l}
0, \frac{3}{4}, 1 \\
0,0,0
\end{array}\right)}{\Gamma\left(\frac{1}{4}\right)} \\
& \psi_{1}\left(\frac{1}{5}\right)=\frac{8 \pi^{2}}{5-\sqrt{5}}-\psi_{1}\left(\frac{4}{5}\right) \\
& \psi_{1}\left(\frac{1}{6}\right)=4 \pi^{2}-\psi_{1}\left(\frac{5}{6}\right) \\
& \psi_{1}\left(\frac{1}{8}\right)=\frac{4 \pi^{2}}{2-\sqrt{2}}-\psi_{1}\left(\frac{7}{8}\right)
\end{aligned}
$$

where $G_{t, u}^{r, s}\left(x \mid \begin{array}{l}a, b, c \\ d, e, f\end{array}\right)$ denotes the Meijer G- function and $\Gamma(x)$ denotes the gamma function.

Using the Theorem 1, I calculate

$$
\begin{gathered}
\psi_{1}\left(\frac{1}{4}\right)=8 C+\pi^{2} \\
\psi_{1}\left(\frac{1}{6}\right)=8 \pi^{2}+\frac{9}{4} \psi_{1}\left(\frac{1}{3}\right)-\frac{3}{2} \psi_{1}\left(\frac{2}{3}\right)-\frac{1}{4} \psi_{1}\left(\frac{5}{6}\right), \\
\psi_{1}\left(\frac{1}{8}\right)=32 C+4 \pi^{2}-\psi_{1}\left(\frac{5}{8}\right)
\end{gathered}
$$

where $C$ denotes the Catalan constant.

From (12) and (16), it follows that

$$
G_{3,3}^{3,2}\left(1 \mid \begin{array}{c}
0, \frac{3}{4}, 1 \\
0,0,0
\end{array}\right)=\Gamma\left(\frac{1}{4}\right)\left(8 C+\pi^{2}\right)
$$

From (14) and (17), it follows that

$$
4 \pi^{2}-\frac{3}{4} \psi_{1}\left(\frac{5}{6}\right)=8 \pi^{2}+\frac{9}{4} \psi_{1}\left(\frac{1}{3}\right)-\frac{3}{2} \psi_{1}\left(\frac{2}{3}\right)
$$

From (11) and (20), it follows that

$$
5 \psi_{1}\left(\frac{2}{3}\right)-\psi_{1}\left(\frac{5}{6}\right)=\frac{28}{3} \pi^{2} .
$$

From (15) and (18), it follows that

$$
\psi_{1}\left(\frac{5}{8}\right)-\psi_{1}\left(\frac{7}{8}\right)=32 C+4 \pi^{2}-\frac{4 \pi^{2}}{2-\sqrt{2}} .
$$

NOTE. I leave the reader to prove the following identities

$$
\psi_{1}(s)=\int_{0}^{\infty} \frac{(u+1)^{-s} \ln (u+1)}{u} d u=\frac{{ }_{3} F_{2}(1, s, s ; s+1, s+1 ; 1)}{s^{2}}, \quad(\Re(s)>0),
$$




$$
\begin{aligned}
& \int_{0}^{\infty}\left[\frac{(u+1)^{-s} \ln (u+1)}{u}\right]^{2} d u \\
& =2 \frac{{ }_{4} F_{3}(2,2 s+1,2 s+1,2 s+1 ; 2 s+2,2 s+2,2 s+2 ; 1)}{(2 s+1)^{3}},\left(\mathfrak{R}(s)>-\frac{1}{2}\right) \\
& \int_{0}^{\infty}\left[\frac{(u+1)^{-s} \ln (u+1)}{u}\right]^{3} d u \\
& =6 \frac{{ }_{5} F_{4}(3,3 s+2,3 s+2,3 s+2,3 s+2 ; 3 s+3,3 s+3,3 s+3,3 s+3 ; 1)}{(3 s+2)^{4}} \\
& \left.\int_{0}^{\infty}\left[\frac{(u+1)^{-s} \ln (u+1)}{u}\right]^{4} d u \quad(s)>-\frac{2}{3}\right) \\
& =24 \frac{{ }_{6} F_{5}(4,4 s+3,4 s+3,4 s+3,4 s+3,4 s+3 ; 4 s+4,4 s+4,4 s+4,4 s+4,4 s+4 ; 1)}{(4 s+3)^{5}} \\
& \left(\mathfrak{R}(s)>-\frac{3}{4}\right)
\end{aligned}
$$

for $n \in \mathbb{N}$

$$
\begin{aligned}
& \int_{0}^{\infty}\left[\frac{(u+1)^{-s-1} \ln (u+1)}{u}\right]^{n} d u \\
& =n ! \frac{n+2 F_{n+1}\left(n, \frac{n s+(n-1), \ldots, n s+(n-1)}{(n+1) \text { times }} ; \frac{n s+n, \ldots, n s+n}{(n+1) \text { times }} ; 1\right)}{(n s+(n-1))^{n+1}} \text {, } \\
& \left(\mathfrak{R}(s)>-\frac{n-1}{n}\right) \text {, } \\
& \int_{0}^{\infty} \frac{(u+1)^{-s-1} \ln (u+1)}{u} d u=\psi_{1}(s+1) \\
& =\psi_{1}(s)-\frac{1}{s^{2}}=\frac{{ }_{3} F_{2}(1, s+1, s+1 ; s+2, s+2 ; 1)}{(s+1)^{2}}, \quad(\Re(s)>-1), \\
& \int_{0}^{\infty}\left[\frac{(u+1)^{-s-1} \ln (u+1)}{u}\right]^{2} d u \\
& =2 \frac{{ }_{4} F_{3}(2,2 s+3,2 s+3,2 s+3 ; 2 s+4,2 s+4,2 s+4 ; 1)}{(2 s+3)^{3}}, \quad\left(\mathfrak{R}(s)>-\frac{3}{2}\right), \\
& \int_{0}^{\infty}\left[\frac{(u+1)^{-s-1} \ln (u+1)}{u}\right]^{3} d u \\
& =6 \frac{{ }_{5} F_{4}(3,3 s+5,3 s+5,3 s+5,3 s+5 ; 3 s+6,3 s+6,3 s+6,3 s+6 ; 1)}{(3 s+5)^{4}}, \\
& \left(\mathfrak{R}(s)>-\frac{5}{3}\right)
\end{aligned}
$$




$$
\begin{aligned}
& \int_{0}^{\infty}\left[\frac{(u+1)^{-s-1} \ln (u+1)}{u}\right]^{4} d u \\
& =24 \frac{{ }_{6} F_{5}(4,4 s+7,4 s+7,4 s+7,4 s+7,4 s+7 ; 4 s+8,4 s+8,4 s+8,4 s+8,4 s+8 ; 1)}{(4 s+7)^{5}}, \\
& \left(\Re(s)>-\frac{7}{4}\right)
\end{aligned}
$$

for $n \in \mathbb{N}$

$$
\begin{aligned}
& \int_{0}^{\infty}[\left.\frac{(u+1)^{-s-1} \ln (u+1)}{u}\right]^{n} d u \\
& \quad=n ! \frac{n+2 F_{n+1}\left(n, \frac{n s+(2 n-1), \ldots, n s+(2 n-1)}{(n+1) \text { times }} ; \frac{n s+(2 n), \ldots, n s+(2 n)}{(n s+(2 n-1))^{n+1}} ; 1\right)}{(n+1) \text { times }} \\
& \quad\left(\mathfrak{R}(s)>-\frac{2 n-1}{n}\right)
\end{aligned}
$$

and for $n \in \mathbb{N}$

$$
\begin{aligned}
& \int_{0}^{\infty}\left[\frac{(u+1)^{-s-j} \ln (u+1)}{u}\right]^{n} d u \\
& =n ! \frac{n+2 F_{n+1}\left(n, \frac{n s+((j+1) n-1), \ldots, n s+((j+1) n-1)}{(n+1) \operatorname{times}} ; \frac{n s+((j+1) n), \ldots, n s+((j+1) n)}{(n s+((j+1) n-1))^{n+1}} ; 1\right)}{(n+1) \text { times }}, \\
& \left(\Re(s)>-\frac{(j+1) n-1}{n}\right) .
\end{aligned}
$$

\section{REFERENCES}

[1] http://en.wikipedia.org/wiki/Trigamma_function, available in March 11, 2014.

[2] Guedes, Edigles, The Natural Logarithm Function and its Applications, to appear. 